TecnoLógicas

ISSN-p 0123-7799

ISSN-e 2256-5337

Vol. 23, No. 47, pp. 1-21

Enero-abril de 2020

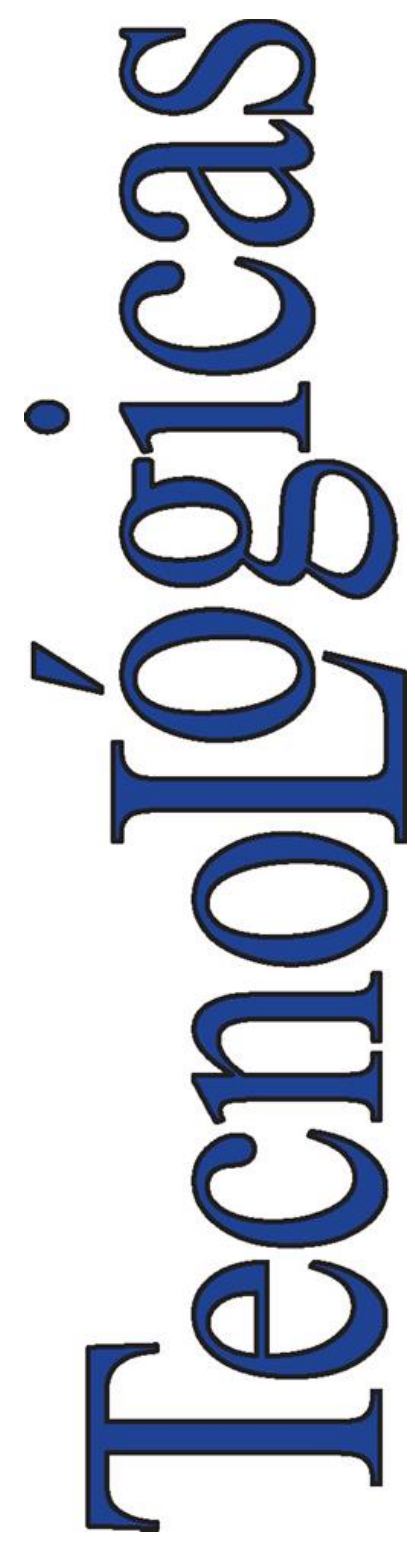

(c) Instituto Tecnológico Metropolitano Este trabajo está licenciado bajo una Licencia Internacional Creative Commons Atribución (CC BY-NC-SA)

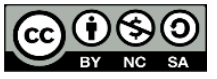

Artículo de investigación/Research Article

\section{Análisis experimental del desempeño de un sistema solar fotovoltaico con inversor centralizado y con microinversores: caso de estudio Manizales}

\section{Experimental Analysis of the Performance of a PV System with a Centralized Inverter and Microinverters: A Study Case in Manizales}

\author{
Claudia L. Cortés (iD) ${ }^{1}$, Gloria S. Gómez-Gómez (iD2, \\ Felipe Betancur-Londoño (iD 3 , \\ Sandra X. Carvajal-Quintero ${ }^{(D)}{ }^{4} \mathrm{y}$ \\ Neil Guerrero-González (D) 5
}

Recibido: 27 de junio de 2019

Aceptado: 12 de septiembre de 2019

Cómo citar / How to cite

C. L. Cortés, G. S. Gómez-Gómez, F. Betancur-Londoño, S. X. CarvajalQuintero, N. Guerrero-González, "Análisis experimental del desempeño de un sistema solar fotovoltaico con inversor centralizado y con microinversores: caso de estudio Manizales", TecnoLógicas, vol. 23, no. 47 , pp. 1-21, 2020. https://doi.org/10.22430/22565337.1403

1 MSc. en Automatización Industrial, Departamento de Ingeniería Eléctrica, Electrónica y Computación, Universidad Nacional de Colombia, ManizalesColombia,clcortesc@unal.edu.co

2 Estudiante Ingeniería Electrónica, Departamento de Ingeniería Eléctrica, Electrónica y Computación, Universidad Nacional de Colombia, ManizalesColombia, gsgomezg@unal.edu.co

3 Estudiante Ingeniería Eléctrica, Departamento de Ingeniería Eléctrica, Electrónica y Computación, Universidad Nacional de Colombia, ManizalesColombia, fbetancurl@unal.edu.co

4 PhD en Ingeniería Línea Automática, Departamento de Ingeniería Eléctrica, Electrónica y Computación, Universidad Nacional de Colombia, ManizalesColombia, sxcarvajalq@unal.edu.co

5 PhD. en Fotónica, Departamento de Ingeniería Eléctrica, Electrónica y Computación, Universidad Nacional de Colombia, Manizales-Colombia, nguerrerog@unal.edu.co 


\title{
Resumen
}

La generación de electricidad amigable con el medio ambiente es un factor fundamental para el crecimiento económico y social de cualquier país. Recientemente la instalación de sistemas de generación fotovoltaicos se ha incrementado a nivel local, aunque su rendimiento depende del lugar de instalación y se ve afectado por diversos parámetros ambientales como la radiación, la temperatura y la precipitación. En el presente trabajo se lleva a cabo un análisis experimental del rendimiento, en términos de potencia generada, de un sistema solar fotovoltaico con inversor centralizado y con microinversores, instalado en la ciudad de Manizales, ubicada a $2.150 \mathrm{~m}$ s.n.m., temperatura promedio de $16.4{ }^{\circ} \mathrm{C} \mathrm{y}$ precipitación de $1.878 \mathrm{~mm}$ al año. Históricamente, este municipio presenta dos periodos de tiempo lluviosos con alta nubosidad entre los meses de abril - junio y octubre - diciembre, y dos periodos de tiempo menos lluviosos entre los meses de enero-marzo y julio-septiembre. Para el experimento se implementó un sistema de generación solar fotovoltaico conformado por seis paneles solares marca Hybrytec con $270 \mathrm{~W}$ de potencia de generación cada uno, y dos sistemas de monitoreo - (i) Wifi - Box ${ }^{\circledR}$ para el arreglo fotovoltaico con inversor centralizado y (ii) EnvoyTM para el arreglo fotovoltaico con microinversores. Los parámetros ambientales recolectados por la estación meteorológica La Nubia, ubicada en los alrededores del sistema solar, y los datos recolectados por los sistemas de monitoreo implementados son analizados en el periodo de tiempo comprendido entre los meses de julio de 2018 y mayo de 2019. Se observa y se concluye que, a pesar de los altos niveles de precipitación promedio acumulada mensual (entre $71 \mathrm{~mm}$ y $262,2 \mathrm{~mm}$ ) y los altos niveles de nubosidad (nublado o mayormente nublado el $76 \%$ del tiempo), el arreglo fotovoltaico con microinversores presenta un 16,5 \% de energía generada por encima del arreglo con inversor centralizado.

\section{Palabras clave}

Fuentes de energía alternativa; fuentes de energía renovable; arreglo fotovoltaico; radiación solar; precipitación.

\begin{abstract}
Generating electricity in an environment-friendly manner is a key factor for the economic and social growth of any country. Recently, the installation of PV generation systems has increased, although their performance depends on the location and is affected by multiple environmental parameters, such as solar radiation level, temperature, and precipitation. This work presents an experimental analysis, in terms of generated power, of a PV system installed in Manizales using (a) a centralized inverter and (b) microinverters. Manizales (2150 $\mathrm{m}$ a.s.l.; average temperature, $16.4^{\circ} \mathrm{C}$; and annual precipitation, $1878 \mathrm{~mm}$ ) has historically presented two rainy seasons with high levels of cloudiness in April-June and October-December, and two seasons with minimum rains in January-March and JulySeptember. For this experiment, a PV generation system was implemented using six Hybrytec solar panels ranging up to 270 Watts per panel and two monitoring systems: (i) Wifi-Box ${ }^{\circledR}$ for the PV array with a centralized inverter and (ii) EnvoyTM for the PV array with microinverters. The authors employed environmental parameters collected at the meteorological station La Nubia, located in the surroundings of the solar power system, and data gathered by the PV monitoring systems between July 2018 and May 2019. Despite the high levels of monthly accumulated precipitation (between $71 \mathrm{~mm}$ and $262.2 \mathrm{~mm}$ ) and cloudiness (cloudy and mostly cloudy $76 \%$ of the time) in the area, the PV array generated $16.5 \%$ more energy with the microinverters than with the centralized inverter.
\end{abstract}

\section{Keywords}

Alternative energy sources; renewable energy sources; photovoltaic array; solar radiation; precipitation. 
Análisis experimental del desempeño de un sistema solar fotovoltaico con inversor centralizado y con microinversores: caso de estudio Manizales

\section{INTRODUCCIÓN}

Las fuentes no convencionales de energía renovable (FNCER) son formas alternativas de generación energética que aprovechan fuentes de origen renovable como energía hidráulica, eólica, solar, mareomotriz, geotérmica y de las biomasas. A diferencia de las fuentes de generación convencionales, las FNCER permiten la descentralización del sistema de generación y hacen posible la implementación de servicios complementarios o de soporte, a partir de generación distribuida [1].

El modelo energético actual es un sistema eléctrico de potencia (SEP) con estructura centralizada [2] donde la participación de las energías renovables se hace difícil debido a la falta de monitoreo y control del sistema, especialmente en el sistema energético colombiano. Las redes eléctricas inteligentes (SG, Smart Grid por sus siglas en inglés) pretenden transformar el sistema actual en un sistema distribuido, permitiendo la participación en el mercado energético de fuentes de energía renovables y posibilitando el aporte de energía a cualquier agente que esté conectado a la red mediante la creación de microgeneradores. Una red inteligente es aquella que incorpora las tecnologías de la información en el servicio de energía eléctrica en todas las etapas del SEP (generación, transmisión, distribución y consumo) [3], con el fin de minimizar el impacto ambiental, mejorar la eficiencia del sistema, la confiabilidad, el servicio y optimizar los mercados de electricidad, así como reducir los costos [4].

Colombia, localizada en la zona noroccidental de América del Sur a $4^{\circ} 0^{\prime} \mathrm{N}$ y $73^{\circ} 15^{\prime} \mathrm{O}$, por su posición geográfica cuenta con uno de los niveles de radiación solar más altos del mundo, con un promedio diario multianual cercano a $4,5 \mathrm{kWh} / \mathrm{m} 2$ [5], lo que facilita el desarrollo y la aplicación de tecnologías alternativas que complementen el sistema de generación energético nacional, y al mismo tiempo contribuyan a la protección del medio ambiente [6].

Según el Informe Nacional de Competitividad 2018-2019 publicado por el Consejo Privado de Competitividad [7], la participación de las fuentes de generación energética en el país para 2017 se distribuyó entre 69,9 \% hidráulica, 29,2 \% térmica y $0,9 \%$ fuentes eólicas, solares y de cogeneradores. El mismo informe señala que para junio de 2018 el número de proyectos de generación energética con registro vigente fue 543 , de los cuales la mayor participación la tienen los proyectos solares con 371 registros, seguidos de los proyectos hidráulicos con 121 registros. Además de tener la mayor participación en número, los proyectos solares generaron 4.051,0 MW representando el 24,4 \% del total de la capacidad de generación (16.587,5 MW) [7].

Actualmente, el $70 \%$ de proyectos fotovoltaicos registrados son ejecutados por entes privados. Por su ubicación, en primer lugar se encuentran los proyectos fotovoltaicos ubicados en la zona de la costa atlántica por ser una de las zonas donde se presentan altos índices de radiación solar, entre 5 y $6 \mathrm{kWh} / \mathrm{m} 2$ mensual [8]. La ciudad de Bogotá D.C. también se destaca en el desarrollo de proyectos fotovoltaicos por su capacidad económica, a pesar de ser considerada como una zona de baja radiación solar (entre 3.5 y $4.5 \mathrm{kWh} / \mathrm{m} 2$ mensual) [8].

El restante $30 \%$ de proyectos fotovoltaicos registrados son ejecutados por entes gubernamentales, especialmente en Zonas No Interconectadas (ZNI) y comunidades de escasos recursos, destacándose el déficit por parte de las entidades públicas en la inversión concerniente a la expansión de las energías renovables no convencionales, en comparación con países como Alemania y Japón donde se recibe financiación del Estado del orden del $60 \%$ al $80 \%$ para la 
implementación de sistemas fotovoltaicos [9].

En el Plan de Expansión de Referencia de Generación y Transmisión 2017-2031 [10] se plantea que el sistema de generación energético colombiano, en el largo plazo, debe incluir alternativas de generación con tecnologías no convencionales como la generación solar fotovoltaica de gran escala. De acuerdo con el análisis de escenarios del plan, a nivel nacional la participación de las fuentes no convencionales de energía renovable pasaría de menos del $1 \%$ a estar entre el $14 \%$ y el $18 \%$ en 2031 [10]. Como parte fundamental para la exitosa implementación del plan de expansión y la ampliación de la cobertura del servicio de energía, se hace necesaria la implementación de sistemas de monitoreo y caracterización del desempeño de los módulos fotovoltaicos y la incidencia del clima sobre la potencia generada; el control operativo y el análisis de datos (adquisición, monitoreo, control y mediciones) son esenciales para encontrar la ubicación apropiada [11], mejorar el funcionamiento y la confiabilidad $y$, en consecuencia, el rendimiento energético y económico de los sistemas fotovoltaicos [12], [13].

El inversor, como elemento primordial para un sistema solar fotovoltaico, puede ser clasificado según su conexión con los paneles solares en inversor con arreglo string o centralizado y microinversor [14].

Un inversor en arreglo string se conecta a paneles solares en serie donde toda la potencia es sumada; el número de paneles interconectados viene dado por la potencia nominal a la que el inversor está diseñado para funcionar, la cual no debe ser superada. Esta potencia variable a la entrada en corriente continua es convertida en corriente alterna para su posterior aprovechamiento. Un microinversor, por otra parte, se encarga de convertir la corriente continua de un solo panel solar en corriente alterna [15].
Estudios realizados han probado que la configuración del arreglo fotovoltaico y la nubosidad influyen en la potencia generada [16], [17]. En este sentido, el propósito de este artículo es estudiar, de manera comparativa, la influencia de la configuración del arreglo fotovoltaico, el impacto de la nubosidad y las ventajas o desventajas que se presentan cuando se cuenta con un único inversor centralizado, y cuando se trabaja con varios microinversores, durante un período de once meses.

\section{INVERSOR Y MICROINVERSOR: MARCO CONCEPTUAL, REGULATORIO Y TÉCNICO}

Los sistemas de generación fotovoltaicos se pueden clasificar según su funcionamiento en aislados y conectados a la red. Los sistemas fotovoltaicos conectados a la red permiten entregar energía a la red pública contrario a los sistemas fotovoltaicos aislados, los cuales se han pensado principalmente para zonas de difícil acceso no conectadas al sistema interconectado nacional. Un sistema fotovoltaico conectado directamente a la red (ver Fig. 1) se compone de un conjunto de paneles solares e inversor.

De forma general, un convertidor de corriente directa (DC) a corriente alterna (AC) se conoce como inversor. La función de un inversor es cambiar un voltaje de entrada DC a un voltaje simétrico de salida $\mathrm{AC}$, con la magnitud $\mathrm{y}$ frecuencia deseadas [18]. Tanto el voltaje de salida como el de entrada pueden ser fijos o variables. Si se modifica el voltaje de entrada DC y la ganancia del inversor se mantiene constante, es posible obtener un voltaje variable a la salida. Por otra parte, si el voltaje de entrada en DC es fijo y no es controlable, se puede obtener un voltaje de salida variable por medio de métodos que modifiquen la ganancia del inversor [19]. 


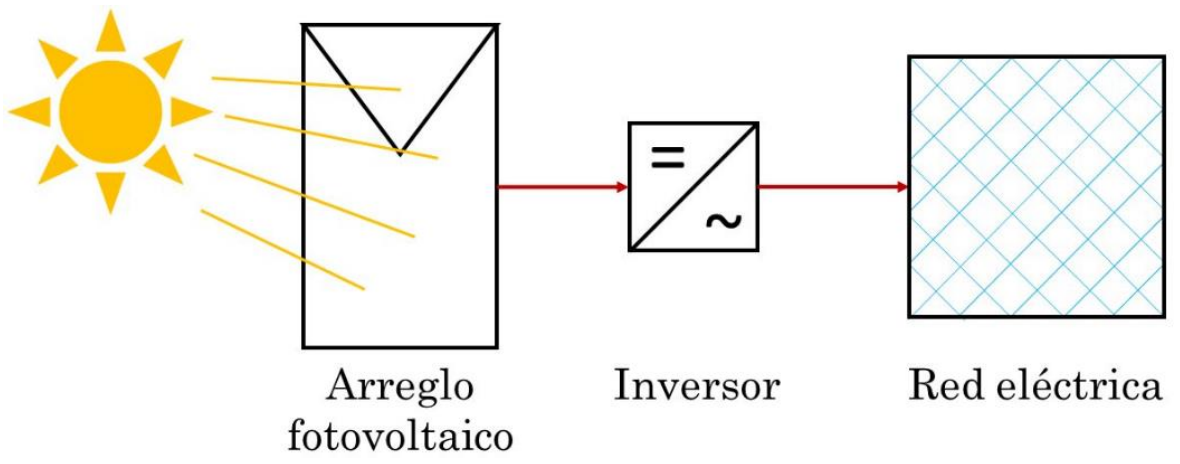

Fig. 1. Sistema fotovoltaico conectado a la red. Fuente: elaboración propia.

El inversor solar es tal vez uno de los elementos más importantes de un sistema fotovoltaico de potencia. Su función es convertir la corriente directa variable de los paneles solares en corriente alterna útil, que puede ser inyectada a la red pública de electricidad o utilizada localmente en un sistema autónomo [20].

A diferencia de los inversores convencionales que están diseñados para operar con una fuente de tensión estable, los inversores para sistemas fotovoltaicos, debido a la naturaleza variable del recurso primario, deben poseer funciones especiales como el control de la potencia generada y protecciones para el arreglo fotovoltaico.

En países desarrollados existen leyes que regulan todos los aspectos técnicos y económicos para la implementación de los sistemas conectados a la red, siendo un tema especial la diferenciación entre inversor centralizado y microinversor, sus límites de potencia y cuándo usar uno $\mathrm{u}$ otro para el diseño del arreglo fotovoltaico.

La operación con inversores centralizados requiere que los paneles solares deban ser conectados en serie, con el fin de formar un solo gran módulo fotovoltaico con tensiones nominales que van desde los 240 VDC a los 1000 VDC. No obstante, este tipo de conexión tiende a tener un desempeño bajo en comparación con la operación de arreglos con microinversores, pues al estar los módulos fotovoltaicos conectados en serie, el desempeño global del sistema puede verse afectado por variaciones mínimas en la potencia generada de alguno de los paneles solares que lo conforma [21].

En este sentido, algunos estudios han destacado que, en condiciones de sombreado ligero, medio e intenso, los arreglos con microinversores han obtenido mejores resultados en cuanto a energía producida en comparación con arreglos string [21].

En cuanto a la operación con microinversores, el prefijo "micro" sugiere que estos inversores están diseñados con el fin de conectarse a un solo panel solar, máximo dos. Aunque no hay un estándar donde se mencionen particularmente los límites de potencia para inversores $\mathrm{y}$ microinversores, para un microinversor la potencia se clasifica en $245 \mathrm{~W}, 400 \mathrm{~W}$ y $500 \mathrm{~W}$ en microinversores duales [22].

En artículos académicos se dan rangos de 100 a $250 \mathrm{~W}$ y hasta $300 \mathrm{~W}$ debido a la eficiencia de los dispositivos [23], [24].

En el comercio los vendedores de estos dispositivos han optado por considerar que los inversores con capacidades menores a $1 \mathrm{~kW}$, en general, son microinversores [25].

La principal ventaja de los microinversores radica en que a cada dispositivo se le asigna su propio panel solar, produciendo energía de manera independiente a los demás paneles del sistema; maximizando la cantidad de potencia generada sin importar las condiciones climáticas, de sombreado y de suciedad presentes en los módulos fotovoltaicos. Adicionalmente, gracias a la 
independencia en la conexión de cada microinversor, este tipo de sistema es escalable $\mathrm{y}$, por ser una tecnología en desarrollo, se realizan constantes estudios con relación al aprovechamiento y la optimización de la potencia generada por medio de técnicas de seguimiento del punto de máxima potencia (MPPT por sus siglas en inglés) [26].

\subsection{Algoritmos para la optimización de la potencia generada}

El objetivo del MPPT (Maximum Power Point Tracking) es encontrar de manera automática el voltaje o la corriente en la cual un arreglo fotovoltaico opera entregando la máxima potencia bajo condiciones de temperatura e irradiancia establecidas, ya que bajo condiciones donde se presentan sombras transitorias se pueden presentar distintos puntos donde se ubican estos niveles máximos [27].

Entre la gran variedad de algoritmos utilizados para MPPT es posible destacar dos que son los más implementados (i) perturbar y observar, (ii) conductancia incremental [27]. El método de perturbar y observar es el más usado debido a su simplicidad de operación, el voltaje en los terminales es constantemente perturbado a la vez que se mide la potencia generada.

Si la potencia aumenta debido a una perturbación en cualquier dirección, indica que el punto de operación se está moviendo hacia el punto de máxima potencia. Por esto, el voltaje se sigue cambiando en la misma dirección en ciclos futuros, hasta que la potencia decrece, indicando que el punto de máxima potencia se ha superado [28].

El método de conductancia incremental tiene una ventaja sobre el de perturbar y observar, ya que calcula la dirección de la perturbación sin estar variando el voltaje en las terminales. Su principio de operación se basa en la curva característica de potencia- voltaje, de esta se deriva una relación entre la corriente y el voltaje y sus razones de cambio, con las cuales se determina la dirección de la perturbación.

Con este algoritmo se puede determinar si el punto de máxima potencia se alcanzó; una vez esto sucede la perturbación se detiene y no se reanuda sino hasta que la relación entre el voltaje y la corriente y sus razones de cambio son diferentes [29].

\subsection{Normas y lineamientos para inversores}

Con el fin de garantizar la seguridad y durabilidad de los equipos utilizados en sistemas solares, las organizaciones internacionales líderes en certificaciones han creado estándares que dictan los requerimientos que deben cumplir estos dispositivos para su manufactura y posterior comercialización. Algunos estándares son de obligatorio cumplimiento, mientras que otros son optativos con el fin de elevar la calidad del producto.

La International Electro-technical Commission (IEC) formuló en 1999 el estándar IEC 61683 para medir la eficiencia de los inversores $y$ acondicionadores de potencia, y a su vez se formuló la norma europea EN 50530 que estandariza la medida general de la eficiencia de los inversores. Por esto los fabricantes de inversores deben especificar en las hojas de datos de sus dispositivos una eficiencia internacional y otra europea.

En cuanto a las especificaciones y requerimientos técnicos para la interconexión de equipos de generación distribuida a nivel internacional se adoptó el estándar IEEE 1547, donde se establecen los criterios referentes al desempeño, operación, ensayos de seguridad y mantenimiento de la interconexión [30], [31], [32].

En Colombia se ha emitido la normativa NTC 5627 donde se establecen los lineamientos para la clasificación y ensayos de componentes de acumulación, conversión y gestión de energía de sistemas fotovoltaicos. Así mismo, se sigue 
la normativa NTC 2183 que aplica a la seguridad de aparatos eléctricos de uso doméstico y el estándar IEEE 1547 [33], [34].

Colombia apenas está dando sus primeros pasos en la integración entre sus sistemas eléctricos tradicionales y los recursos renovables no convencionales, y puede aprender de otras naciones donde la penetración significativa de estos recursos ha llevado a modificaciones costosas, tanto en su infraestructura de transporte y distribución de energía eléctrica, como en el diseño de mercados de servicios auxiliares [35].

Con las modificaciones hechas por estos países pioneros en energías renovables, se ha logrado el desarrollo de mercados integrales de energía, en donde se implementan mecanismos de comercialización y procesos de remuneración que tienen en cuenta el beneficio de generación de los recursos renovables, así como su participación con servicios complementarios que garantizan la calidad y continuidad de la energía eléctrica. Lo anterior, reduce el impacto de las energías renovables en los equipos de distribución, por medio del control del nivel de tensión, entrega de activos y reactivos a la red, así como un mayor compromiso por parte de los operadores de red en cuanto al acompañamiento de los nuevos generadores distribuidos [36].

Todo esto, junto con el desarrollo de normativas en donde se estipulen los requerimientos para el establecimiento de la operación en isla intencional, necesaria en zonas donde la continuidad del servicio es un inconveniente, beneficia no solo al usuario final sino también a las empresas prestadoras del servicio con la reducción de penalizaciones y costos por confiabilidad.

\subsection{Protecciones en los inversores}

De acuerdo con los estándares mencionados anteriormente, es fundamental que los inversores ofrezcan protección contra una serie de fallas que eventualmente se pueden presentar en arreglos fotovoltaicos. Entre las características requeridas se encuentran: protección contra sobretensiones, polaridad inversa, aislamiento galvánico con puesta a tierra, o en sistemas que no cuenten con esta, que tengan una tierra generada por el equipo, deben contar con un sistema de protección contra fallas a tierra $\mathrm{y}$ protección anti-isla para sistemas que inyectan energía a la red pública [37].

Las instalaciones fotovoltaicas generalmente se ubican en sitios como techos y demás lugares al aire libre, esto las hace susceptibles a daños debidos a sobretensiones; por esta razón, los inversores deben incluir en su diseño una forma de mitigar el impacto de las sobretensiones con dispositivos orientados a la generación fotovoltaica, tanto en el lado de entrada DC como en la salida AC. Los inversores usan la electrónica de potencia y su conmutación para generar una onda sinusoidal; por la duración y el periodo de estas conmutaciones en la salida del inversor se pueden superponer picos de tensión, que pueden crear falsas sobretensiones provocando repetidas interrupciones en el funcionamiento del sistema, lo que deteriora a mediano plazo las protecciones convencionales [38].

Con el fin de garantizar la vida útil de los equipos del sistema fotovoltaico, los inversores deben equiparse con un sistema de protección contra polaridades inversas accidentales. Además, los inversores centralizados también se equipan con sistemas que protegen el o los arreglos fotovoltaicos de corrientes inversas, dado que estas pueden alcanzar valores considerables de 10 a 80 Amperios [39].

Especialmente en arreglos fotovoltaicos de gran tamaño, donde se tienen tensiones nominales de entrada de hasta 1 kVDC [39], los módulos que lo conforman no son capaces de soportar estos tipos de corrientes y, en ausencia de un sistema que 
los proteja, desarrollarán fallas a corto plazo.

Prevenir las fallas a tierra es fundamental y requiere de una labor intensiva en sistemas a gran escala; este tipo de fallas pueden volverse peligrosas. En algunos estándares se menciona que los inversores que estén suministrando energía a la red pública se deben desconectar en el evento de una falla a tierra, pero el hecho de desconectar y apagar el equipo no soluciona la falla. Las fallas a tierra representan un riesgo de incendio por cortocircuito y electrocución, ya que el conductor de tierra deja de estar a su potencia habitual por lo que las superficies y estructuras conectadas al conductor de tierra pueden pasar a estar energizadas, representando un riesgo de shock eléctrico al personal técnico.

En ausencia de una protección contra fallas a tierra adecuada en el sistema el riesgo de incendio aumenta, debido a que no hay forma de interrumpir la corriente que comienza a incrementarse en los conductores hasta superar su capacidad. Esta corriente puede llevar a conflagraciones que deriven en la pérdida total de los equipos y las estructuras donde se ubican, especialmente en instalaciones residenciales [40].

Dado que los inversores se conectan a la red pública, los estándares de cada operador de red deben obedecerse en cuanto a calidad de la potencia y pruebas requeridas en la interconexión. El estándar internacional IEEE 1547 estipula los lineamientos para la detección de operación en isla, así como los requerimientos para la operación en isla intencional; los inversores que suministren energía a la red pública deberán tener la habilidad para detectar cuando el sistema quede en condición de isla, con el fin de proteger tanto a personal técnico como a los equipos conectados a la red [41].

En el estándar IEEE 1547.4 se cubre el funcionamiento de generadores distribuidos en isla intencional. La operación en isla se refiere al funcionamiento del sistema fotovoltaico y su inversor o inversores, cuando la red pública ha sido desenergizada a propósito o accidentalmente. A los sistemas en isla con recursos distribuidos se les conoce como microgrids; una microgrid debe tener la capacidad de desconectarse y conectarse con un operador de red que les provea energía. En el estándar IEEE 1547.6 se menciona que el funcionamiento en isla de un recurso distribuido mientras está conectado a la red de distribución desenergizada debe evitarse; en caso de que las protecciones actúen y los generadores distribuidos sigan funcionando, los mismos no tendrán la suficiente capacidad para alimentar las cargas adicionales, por lo que los valores de frecuencia y tensión serán distintos a los nominales, causándoles daños eventualmente. Además, como las protecciones no poseen la capacidad de sincronismo, pero sí de recierre en condición de isla, al momento de una reconexión la tensión de la red utilitaria y el generador distribuido pueden estar desfasadas provocando la reapertura de las protecciones, situación que puede desembocar en daños a los equipos de generación distribuida y a las protecciones [41], [42].

\section{CONFIGURACIÓN EXPERIMENTAL}

Con el fin de realizar un estudio de la potencia generada en un sistema fotovoltaico instalado en la ciudad de Manizales, se implementó un sistema de monitoreo y caracterización del rendimiento, conformado por seis paneles solares de marca Hybrytec, cada uno con una potencia de generación de $270 \mathrm{~W}$, con peso de $18,5 \mathrm{~kg}$ y dimensiones de largo, ancho y profundidad 1640 × 990 × $35 \mathrm{~mm}$ respectivamente; cada panel posee 60 células solares de silicio policristalino de dimensiones 156 x $156 \mathrm{~mm}$ [43]. 
Los datos obtenidos son correlacionados luego con las condiciones climatológicas registradas en la ciudad de Manizales a través de su sistema de monitoreo ambiental. Manizales cuenta con una red telemétrica de 14 estaciones meteorológicas e hidrometeorológicas que miden y transmiten en tiempo real datos de variables climáticas asociadas a la prevención de desastres, y en particular a eventos de precipitación extremos que generan deslizamientos en laderas y taludes de la ciudad [44].

Las estaciones meteorológicas (ver Fig. 2) miden variables asociadas al comportamiento de la atmósfera, en este caso ocho variables entre las que se encuentran temperatura, humedad, precipitación, velocidad del viento $\mathrm{y}$ radiación solar [46].

Los datos recolectados por cada sensor son transmitidos de manera telemétrica vía radioenlace a la estación central, localizada en las instalaciones del Instituto de Estudios Ambientales de la Universidad Nacional, donde se registra, almacena y se procesa en tiempo real cada 5 minutos.
Esta información se puede consultar en un rango de tiempo específico y puede mostrarse de manera simultánea (varias variables) o individual, ya sea diaria, mensual o anual.

La configuración experimental del sistema de monitoreo y caracterización implementado se ubica en los alrededores de la estación meteorológica La Nubia, localizada a $5^{\circ} 1^{\prime} 44.1^{\prime \prime} \mathrm{N}$ y $75^{\circ} 28^{\prime} 20^{\prime \prime} \mathrm{W}$ en el bloque $\mathrm{P}$ del campus La Nubia de la Universidad Nacional.

Los sistemas de generación se clasifican en dos tipos dependiendo de si se encuentran aislados o conectados a la red eléctrica [47]. El sistema fotovoltaico implementado se conecta directamente a la red (ver Fig. 2) y se compone de paneles solares e inversor marca Hersic para el arreglo fotovoltaico en configuración string o microinversores M250 marca Enphase ${ }^{\mathrm{TM}}$. Los dos arreglos fotovoltaicos instalados funcionan de forma paralela para realizar el análisis comparativo en términos de potencia generada $y$ se describen a continuación.

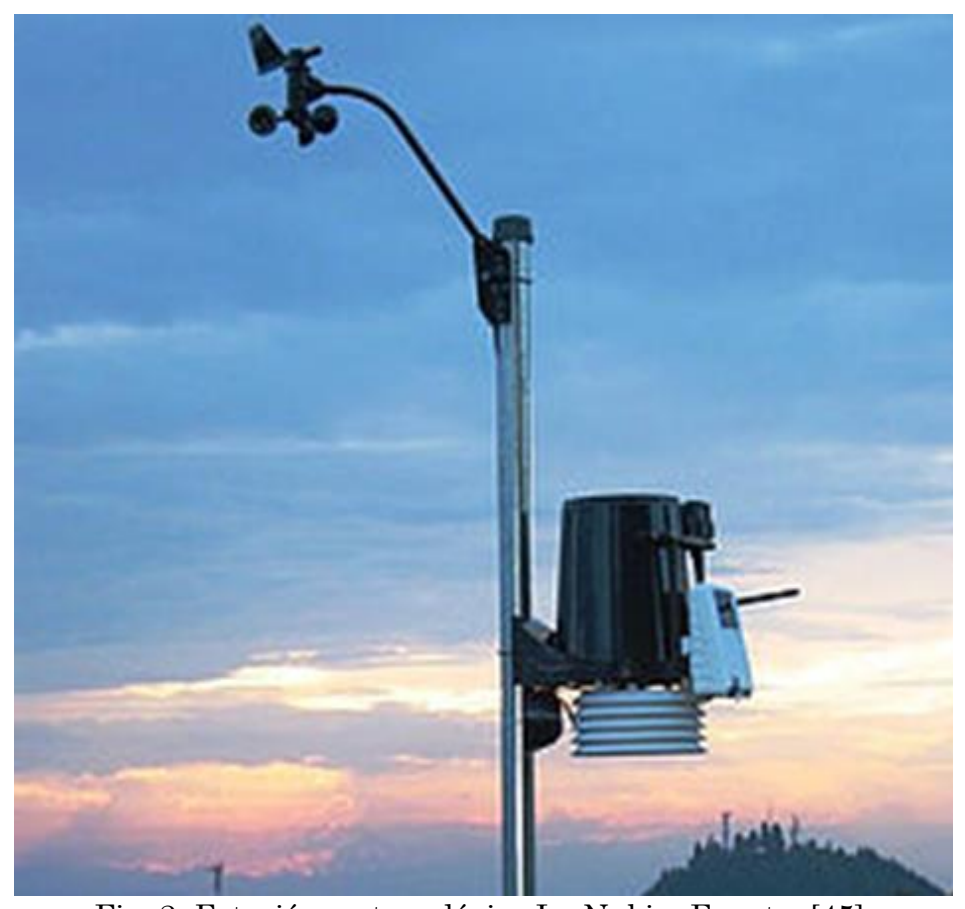

Fig. 2. Estación meteorológica La Nubia. Fuente: [45]. 


\subsection{Sistema microinversores}

fotovoltaico

con

Como se observa en la Fig. 3, el subsistema está compuesto por 3 paneles solares conectados de manera independiente a 3 microinversores M250 Enphase $^{\mathrm{TM}}$ [48].

La interconexión de estos convertidores DC a AC se hace a través del cable Engage $^{\mathrm{TM}}$, del cual se derivan tres ramificaciones que se conectan al tablero de distribución y al sistema de monitoreo Envoy ${ }^{\mathrm{TM}}$.

Los microinversores $\mathrm{M} 250^{\mathrm{TM}}$ poseen una potencia nominal de $240 \mathrm{~W}$ cada uno, son compatibles con módulos de 60 y 72 celdas, tienen enfoque CA completo $\mathrm{y}$ conexión a tierra integrada; cuentan adicionalmente con transformadores de alta frecuencia galvánicamente aislados que ayudan a minimizar el efecto de las sombras, el polvo y la suciedad [48].

\subsection{Sistema fotovoltaico con inversor centralizado}

Como se observa en la Fig. 4, el subsistema está compuesto por 3 paneles solares conectados entre sí mediante cable solar en configuración serial a un inversor marca Hersic.

El inversor Hersic posee una potencia nominal de $1500 \mathrm{~W}$ y una eficiencia máxima del $97 \%$ de conexión a la red, su diseño se caracteriza por no poseer transformador a prueba de agua y polvo, cuenta con tecnología MPPT avanzada (regulador, usa el máximo rendimiento del panel, punto de máxima potencia), sin ventilador y con nivel de ruido menor a $30 \mathrm{~dB}[49]$.

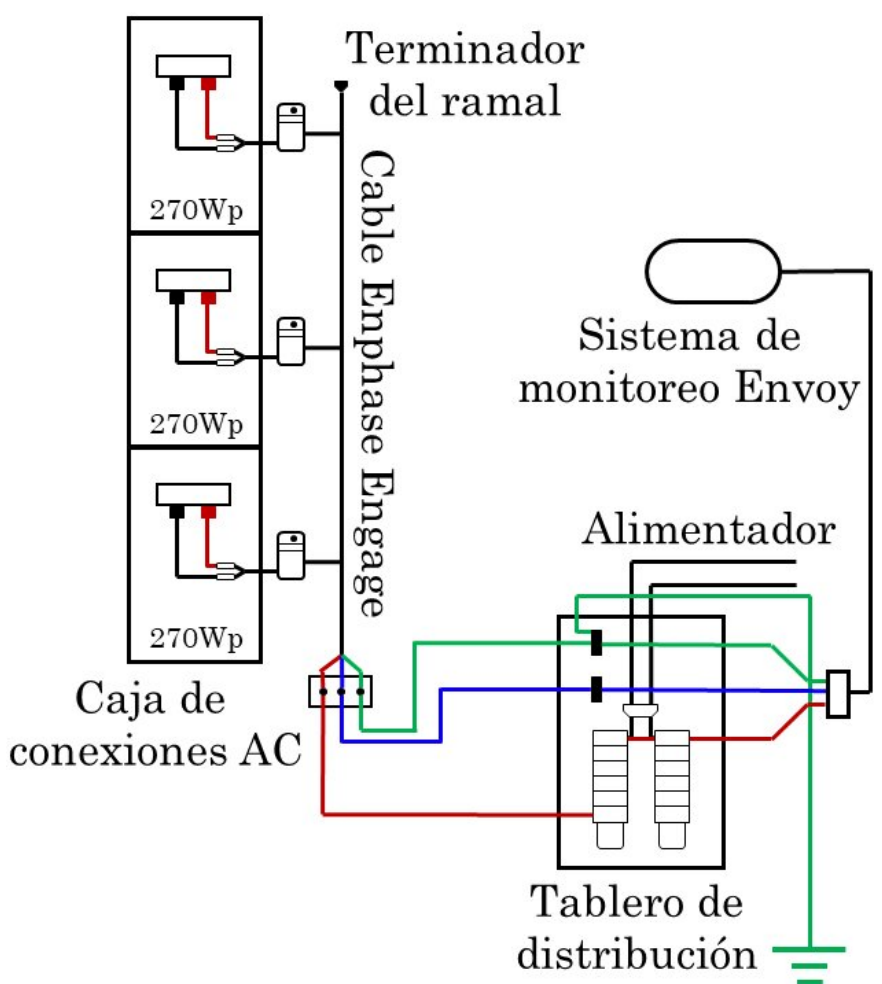

Fig. 3. Diagrama de conexiones del subsistema 1. Fuente: elaboración propia. 


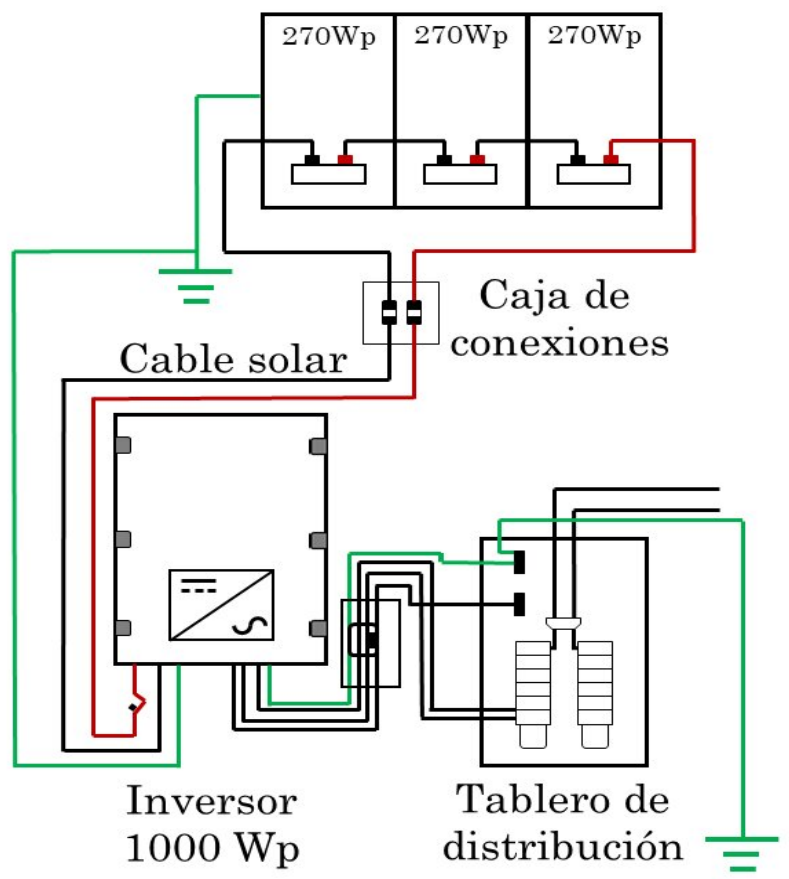

Fig. 4. Diagrama de conexiones del subsistema 2. Fuente: elaboración propia.

\section{METODOLOGÍA}

El experimento se realiza durante 24 horas diarias por once meses entre julio de 2018 y mayo de 2019. Durante este periodo se toman los valores promedios diarios de los indicadores climáticos de la estación más cercana al sistema de generación fotovoltaico (Estación Meteorológica La Nubia). Los índices climáticos de temperatura promedio $\left({ }^{\circ} \mathrm{C}\right)$, radiación solar media $\left(\mathrm{W} / \mathrm{m}^{2}\right)$ y precipitación $(\mathrm{mm})$ diarios y mensuales, fueron usados para analizar el desempeño para cada arreglo fotovoltaico.

La interconexión de los dos arreglos forma un solo sistema de generación (ver Fig. 5) en el que la sumatoria de la potencia de generación de cada uno aporta energía, tanto al suministro eléctrico como a las cargas adaptadas a él.

Para analizar el rendimiento y el desempeño se realizan gráficas comparativas de potencia de generación, con el reporte ponderado por mes de los índices climáticos. Para dicha comparación, la generación total de cada subsistema se calcula con la sumatoria de los datos de producción (energía generada en Wh) de los días por mes de cada subsistema, como se expresa en (1).

$$
\begin{aligned}
P_{\text {total-mes }} & =\sum_{i=1}^{N} \text { generación }[W h] \\
\therefore i & =\# \text { días del mes }
\end{aligned}
$$

Cada arreglo fotovoltaico posee un sistema independiente para la recolección de datos. El arreglo con microinversores utiliza el sistema Envoy ${ }^{\mathrm{TM}}$ y el software de supervisión y análisis de Enphase Enlighten $^{\mathrm{TM}}$, el cual envía información vía wifi a la aplicación móvil MyEnlighten $^{\mathrm{TM}}$ [50]; su configuración permite ver el estado o la descripción de patrones de energía en el día, mes, año o durante la vida útil del sistema.

El tipo de información que recolecta y muestra cada 15 minutos, se almacena y se descarga en un archivo CVS; entre las variables recolectadas por el sistema se encuentran energía generada, energía consumida, energía exportada a la red e importada de la red, medidas en Wh. 


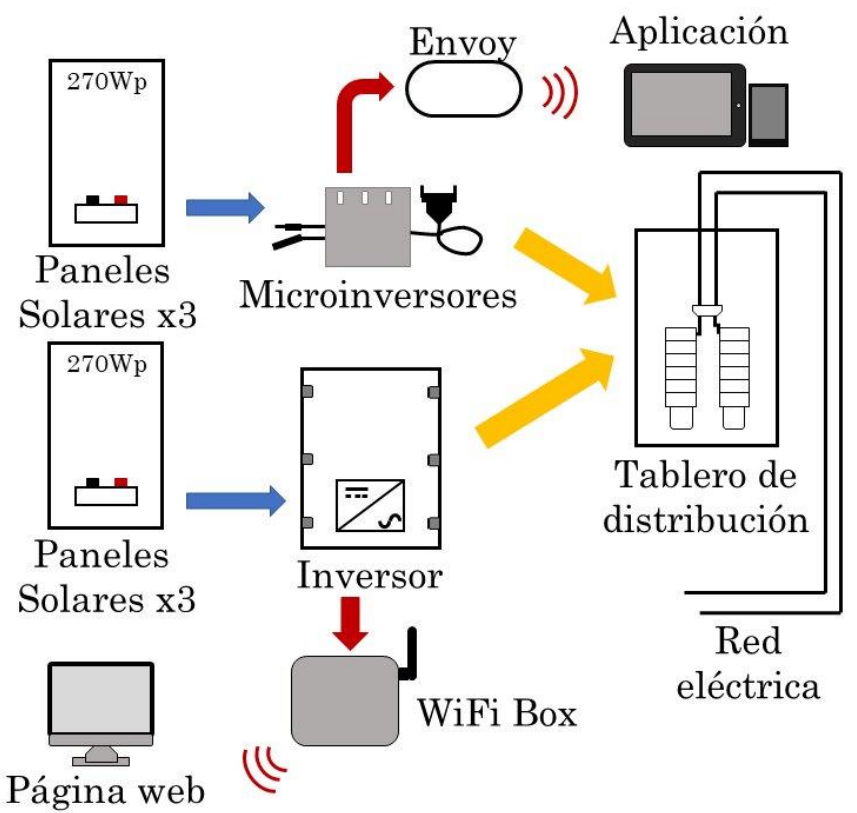

Fig. 5. Interconexión de los subsistemas. Fuente: elaboración propia.

Por su parte el sistema wifi - Box ${ }^{\circledR}$ permite el envío de información recolectada del arreglo con inversor centralizado a una página web; el sitio web muestra el reporte de generación energética medida en $\mathrm{kWh}$ de cada día del mes y permite ser descargado en un archivo XLS.

La conexión entre cada parte del sistema se realiza mediante cables especializados, que garantizan un buen acoplamiento y un mínimo de pérdidas eléctricas de un punto a otro. Esta buena conexión hace que los datos obtenidos por cada sistema de recolección sean confiables y de alta calidad.

\section{RESULTADOS Y DISCUSIÓN}

La generación mensual durante once meses para el sistema fotovoltaico con microinversores se presenta en la Fig. 6.

La comparación con los indicadores climáticos y la energía que produce la configuración con microinversores depende en gran medida de la radiación solar; como se observa en la Fig. 6, se presenta un máximo de generación en el mes de agosto de 2018 de $76,6 \mathrm{kWh} /$ mes y un mínimo de $48,4 \mathrm{kWh} /$ mes en el mes de mayo de 2019.

En la Fig. 7 se presenta la generación mensual durante el mismo periodo de tiempo para el sistema fotovoltaico con inversor centralizado.

La energía máxima total de generación de la configuración con inversor centralizado tiene un comportamiento similar a la configuración con microinversores, como se observa en la Fig. 7, presentando un valor máximo de $69,4 \mathrm{kWh} / \mathrm{mes}$ en el mes de agosto de 2019 y un mínimo de $34,5 \mathrm{kWh} / \mathrm{mes}$ en el mes de mayo de 2019.

En la Fig. 8 se presenta en detalle la comparación de los valores mensuales de generación de los arreglos fotovoltaicos con microinversores y con inversor centralizado.

Para las mismas condiciones climáticas, la generación total acumulada durante los once meses de prueba fue dada en mayor cantidad por el arreglo fotovoltaico con microinversores (691 kWh) en comparación con el arreglo con inversor centralizado (593,2 kWh), como se puede calcular según los valores en la Fig. 8. 


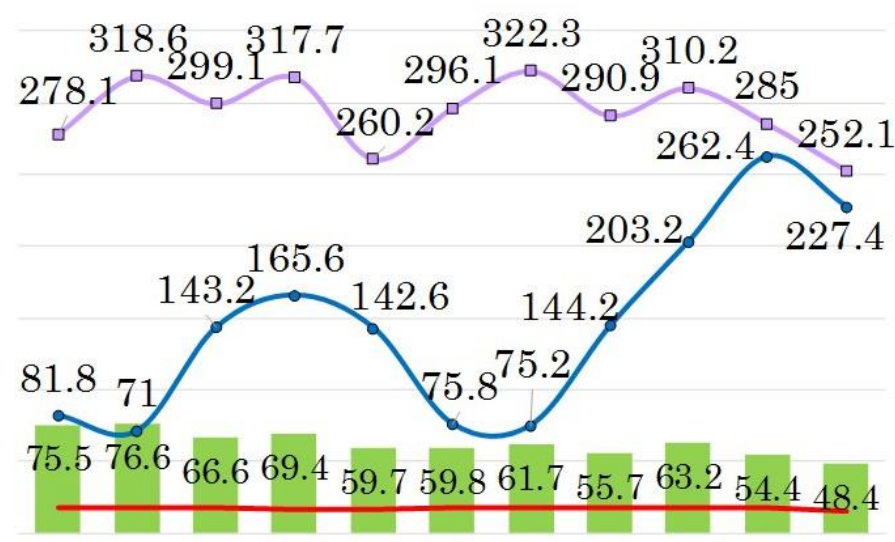

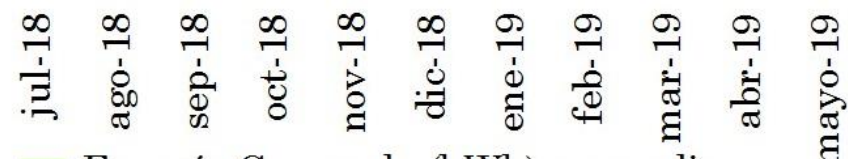

Energía Generada (kWh) promedio

- - Radiación Solar (W/m2) promedio

$\rightarrow$ Precipitación ( $\mathrm{mm}$ ) acumulada

- Temperatura $\left(15,2^{\circ} \mathrm{C}-18,2^{\circ} \mathrm{C}\right)$ promedio

Fig. 6. Comparación índices climáticos con energía generada para el sistema fotovoltaico con microinversores. Fuente: elaboración propia.

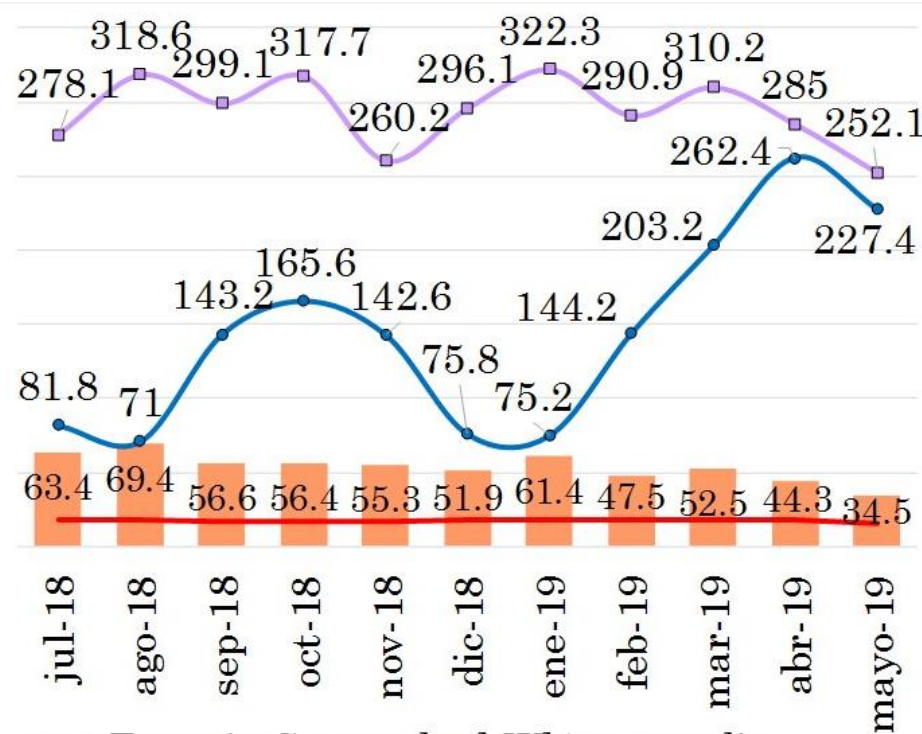

Energía Generada $(\mathrm{kWh})$ promedio

- -Radiación Solar (W/m2) promedio

$\rightarrow$ Precipitación (mm) acumulada

- Temperatura $\left(15,2^{\circ} \mathrm{C}-18,2^{\circ} \mathrm{C}\right)$ promedio

Fig. 7. Comparación índices climáticos con energía generada para el sistema fotovoltaico con inversor centralizado. Fuente: elaboración propia. 


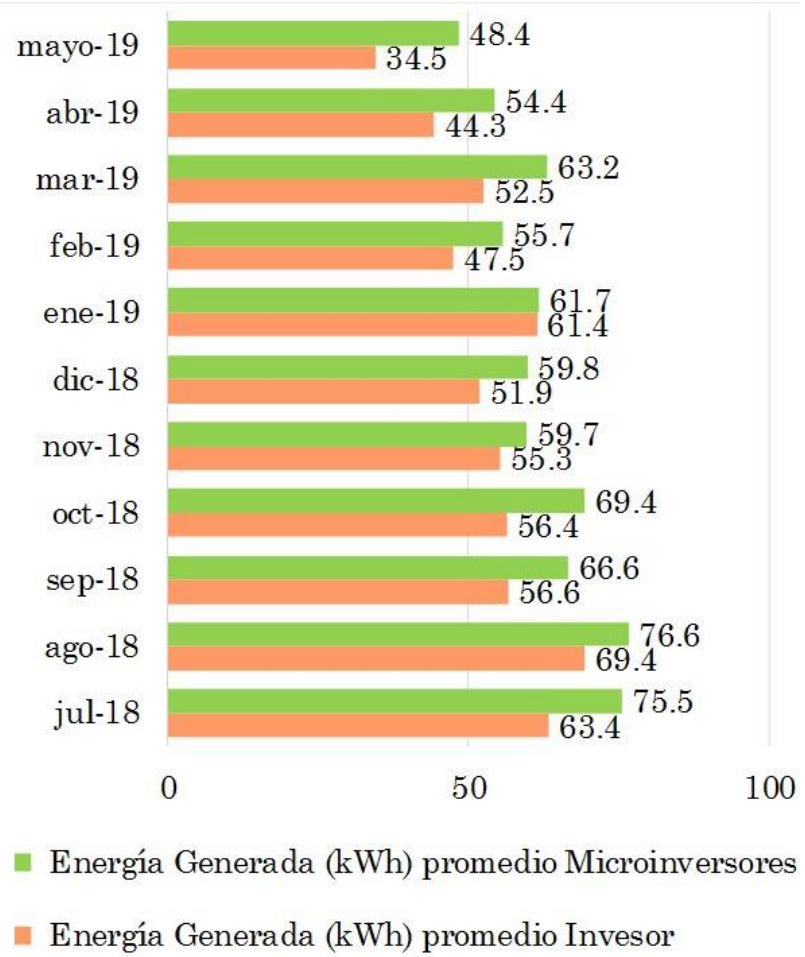

Fig. 8. Comparación datos de generación de ambos subsistemas. Fuente: elaboración propia.

Según reportes de la Unidad de Planeación Minero Energética (UPME), para el año 2018 el consumo per cápita de energía de un colombiano fue de aproximadamente $1.159 \mathrm{kWh}$ por año, de los cuales $499 \mathrm{kWh}$ pertenecen al sector residencial. Según este reporte, para los once meses de prueba la generación de energía total dada por el arreglo fotovoltaico con microinversores o con inversor centralizado cubren esta demanda. Si se considera un hogar compuesto por cuatro integrantes, la generación total dada por el arreglo fotovoltaico con microinversores representa aproximadamente el 34,62 \% del consumo per cápita de energía de un hogar y el $29,72 \%$ para el arreglo con inversor centralizado. Despreciando los costos asociados a la adquisición e instalación del sistema fotovoltaico, estos porcentajes se pueden entender como un ahorro en el pago por el servicio de energía eléctrica en el hogar.

En cuanto a la operación del sistema, los once meses de funcionamiento del sistema fotovoltaico instalado en techo suman 335 días de registro.

Con el propósito de visualizar la frecuencia en generación, en la Fig. 9 se presenta el histograma comparativo entre los arreglos fotovoltaicos con inversor centralizado y con microinversores.

El histograma permite observar que para ambos arreglos fotovoltaicos es más frecuente que la generación diaria oscile entre $1 \mathrm{kWh}$ y $2 \mathrm{kWh}$ : para el arreglo fotovoltaico con inversor centralizado 171 días, el $51 \%$ de días registrados, mientras para el arreglo fotovoltaico con microinversores 153 días, el 45,7\% de días registrados. 
Análisis experimental del desempeño de un sistema solar fotovoltaico con inversor centralizado y con microinversores: caso de estudio Manizales

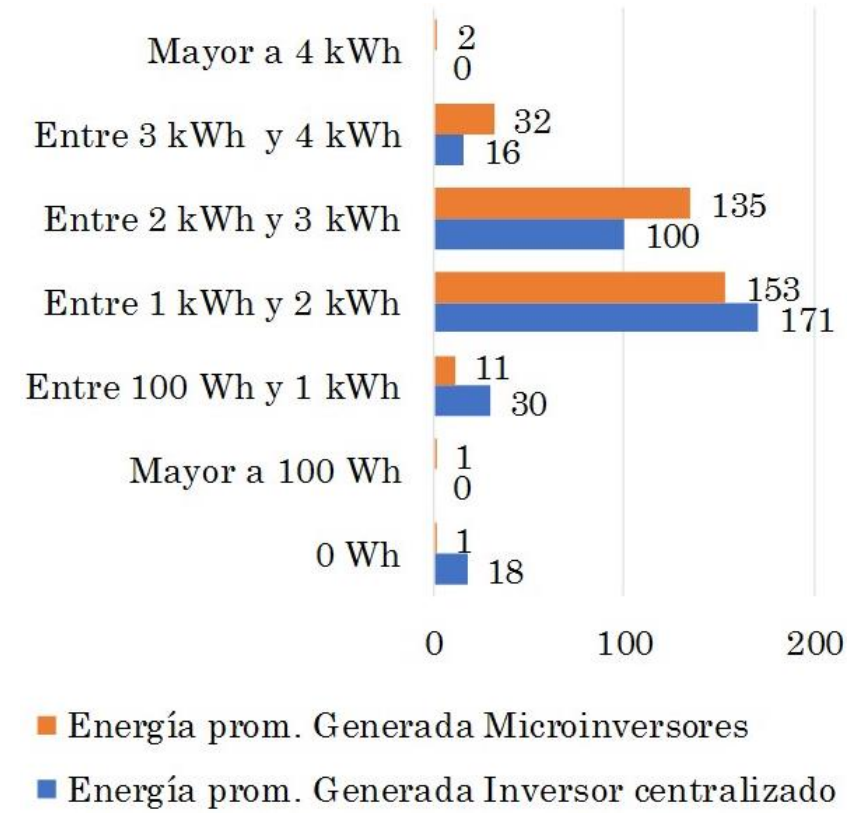

Fig. 9. Histograma comparativo ambos subsistemas. Fuente: elaboración propia.

Se observa, adicionalmente, con los valores extremos del histograma que el arreglo fotovoltaico con microinversores presenta mejor desempeño, registra 2 días con una generación mayor a $4 \mathrm{kWh}$ y solo 1 día no registra generación, mientras el arreglo fotovoltaico con inversor centralizado no registra días con generación mayor a $4 \mathrm{kWh}$ y 18 días no registra generación.

La información detallada por meses se puede observar en la Fig. 10 para el arreglo fotovoltaico con inversor centralizado y en la Fig. 11 para el arreglo fotovoltaico con microinversores.

El histograma mensual de generación de cada arreglo permite conocer los rangos de generación promedio diaria obtenidos durante los once meses de registro. Por ejemplo, para los meses de julio y agosto de 2018 donde la energía promedio generada superó los $63 \mathrm{kWh}$ se registraron los únicos dos días con generación por encima de los $4 \mathrm{kWh}$ para el arreglo fotovoltaico con microinversores.

Se observa además que para el arreglo con inversor centralizado los 18 días de registro con energía promedio generada cero se presentan en 9 de los once meses de registro, especialmente en los meses de octubre y diciembre de 2018, con 4 y 5 días con registro en cero respectivamente. A pesar de los continuos registros en cero ninguno de estos meses presenta un valor de energía promedio generada menor al registrado para el mes de mayo de 2019 en ninguno de los dos arreglos fotovoltaicos evaluados.

Teniendo en consideración los valores de energía promedio generada para los arreglos fotovoltaicos, se realiza un análisis de las variables climáticas para los meses de mayor y menor generación registrados; en la Fig. 12 se presenta el histograma de la precipitación para los meses de agosto de 2018 y abril de 2019.

Para el mes de agosto de 2018 se registró el menor valor de precipitación acumulada $(71 \mathrm{~mm})$ mientras el mes de abril de 2019 registró el segundo valor mayor de precipitación acumulada (213.6 mm).

El mes de agosto de 2018 se caracterizó por presentar lluvias diarias menores a 20 mm mientras para el mes de mayo de 2019 se presentaron incluso lluvias diarias mayores a $30 \mathrm{~mm}$ con un máximo de $43 \mathrm{~mm}$ registrados. 
Análisis experimental del desempeño de un sistema solar fotovoltaico con inversor centralizado y con microinversores: caso de estudio Manizales

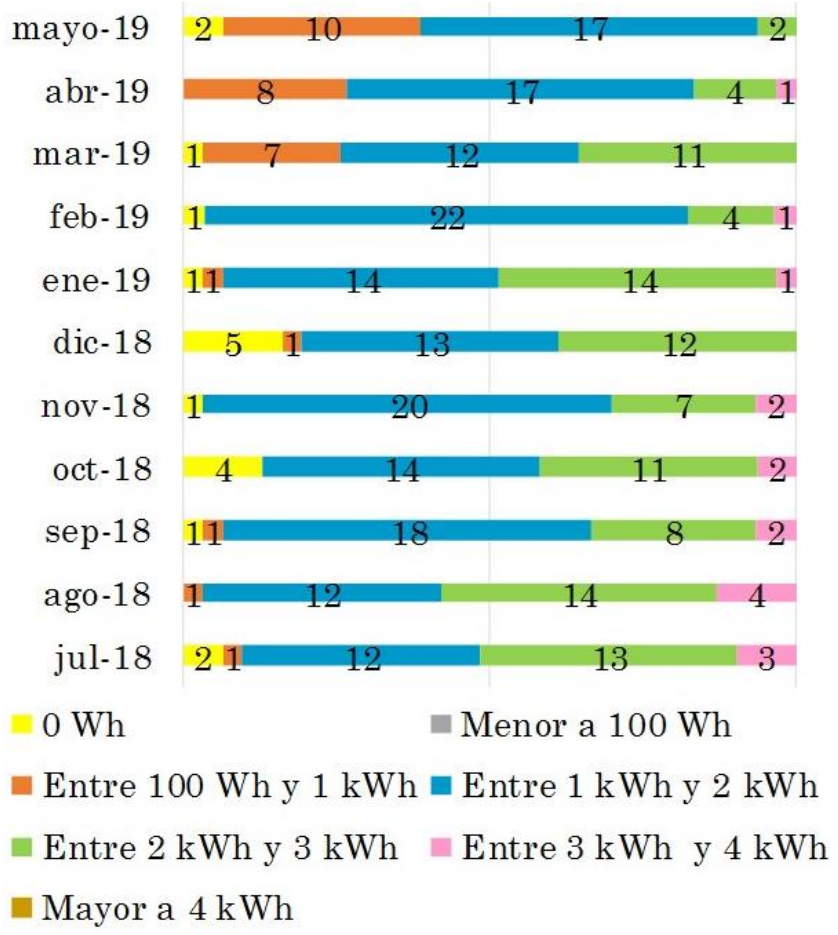

Fig. 10. Histograma mensual de generación arreglo fotovoltaico con inversor centralizado. Fuente: elaboración propia.

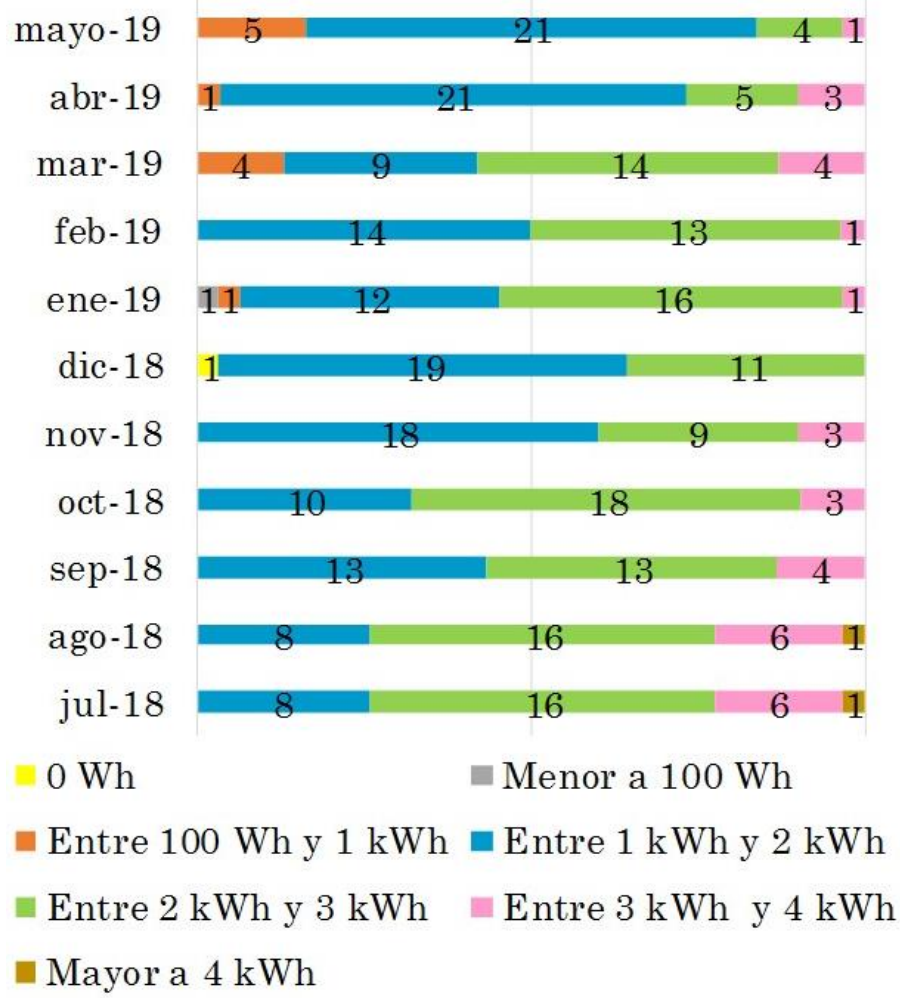

Fig. 11. Histograma mensual de generación arreglo fotovoltaico con microinversores. Fuente: elaboración propia. 


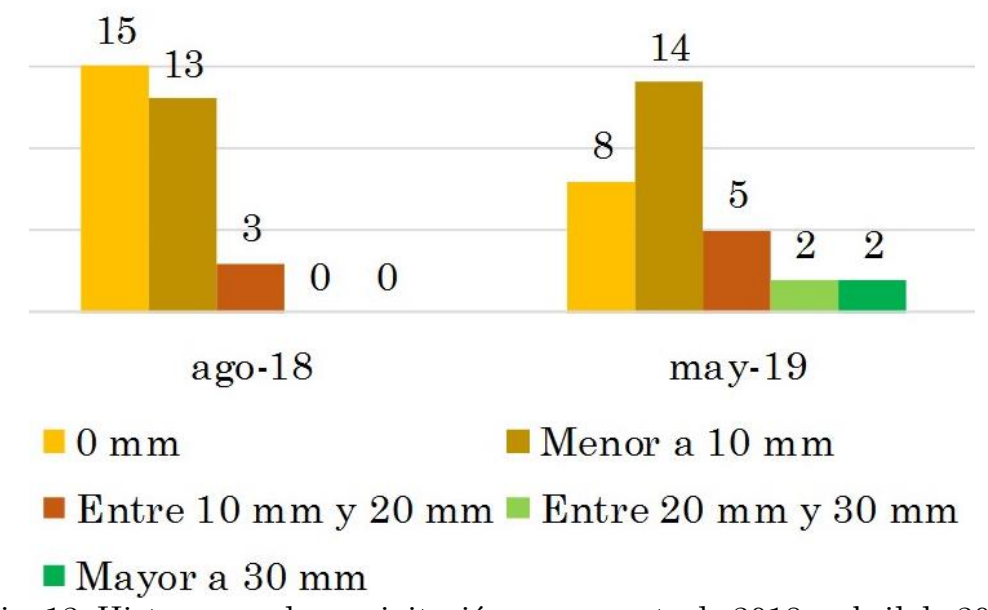

Fig. 12. Histograma de precipitación para agosto de 2018 y abril de 2019 Fuente: elaboración propia.

\section{CONCLUSIONES}

En el presente artículo se llevó a cabo un análisis experimental del rendimiento, en términos de potencia generada, de un sistema solar fotovoltaico con inversor centralizado y con microinversores instalado en la ciudad de Manizales.

Como se ha estudiado ampliamente, la diferencia de energía generada entre los arreglos fotovoltaicos con inversor centralizado y con microinversores es significativa. Cada arreglo fotovoltaico, compuesto por 3 paneles solares, con una potencia de generación de $270 \mathrm{~W}$ cada uno, se instaló en techo. Para once meses de pruebas, el arreglo con microinversores presentó un 16,5\% adicional en energía generada, comparado con el arreglo con inversor centralizado.

Este tipo de experimentos se han realizado anteriormente y se conocen como la cuantificación del efecto mismatching o efecto sombra [51], [52]. En la literatura se encuentran porcentajes de ganancia que no supera el $12 \%$. Dichos experimentos se han realizado en el laboratorio usando simuladores. Como se mencionó anteriormente, en el caso de estudio del artículo los resultados obtenidos fueron de $16,5 \%$, usando un arreglo fotovoltaico instalado en la ciudad de Manizales.
La ciudad de Manizales es una ciudad con alta nubosidad [53], sin embargo, la ciudad se encuentra enclavada en la media montaña andina tropical, sujeta a la acción de los vientos Alisios del noreste y del sureste que definen la zona de confluencia intertropical (ZCIT) [54], [55]; dichas corrientes de vientos favorecen el desplazamiento repentino de nubes [56].

Otro factor que favorece el efecto de mismatching es la suciedad que se acumula en los paneles solares. Sin embargo, la alta precipitación de lluvias en la región asegura una limpieza permanente de los sistemas de generación solar fotovoltaica, ya que el promedio histórico de pluviosidad supera los 2000 $\mathrm{mm}$ acumulados al año [55].

Las anteriores características geográficas y climáticas de la ciudad donde se realizó el experimento muestran que el efecto mismatching no es constante durante los meses evaluados y esto se debe a las condiciones variables de la nubosidad $\mathrm{y}$ al alto nivel de precipitaciones en la ciudad de Manizales.

En este documento también se analizó los factores externos que afectan la eficiencia de los paneles solares, variaciones climáticas como radiación, precipitación y temperatura del ambiente.

La temperatura ambiente también altera la eficiencia del sistema solar 
Análisis experimental del desempeño de un sistema solar fotovoltaico con inversor centralizado y con microinversores: caso de estudio Manizales

fotovoltaico; en la literatura se encuentran evidencias de que los sistemas solares fotovoltaicos disminuyen la eficiencia luego de que la temperatura ambiente supere los 24 grados centígrados [57]-[61].

Aunque los expertos meteorológicos resaltan que, por las condiciones variables de viento y nubosidad, Manizales cuenta con 8 microclimas y dado la ubicación exacta del experimento, la temperatura corresponde a los datos de la estación meteorológica de La Nubia, el reporte del semestre muestra que las temperatura se mantuvo entre 12 a $23{ }^{\circ} \mathrm{C}$ [62].

En Manizales la generación de energía con sistemas fotovoltaicos se ve fuertemente afectada por el clima de montaña, especialmente por la nubosidad presente en la ciudad que, para el caso de este estudio, se asocia directamente a la precipitación registrada por la estación meteorológica instalada en las inmediaciones del sistema fotovoltaico.

Del mismo modo se encuentra que, aunque la variación de la radiación es el efecto más notorio para reducir la producción de energía de un panel, en este caso no es un factor climático determinante; la radiación solar en la ciudad varía aproximadamente entre 260 a $322 \mathrm{~W} / \mathrm{m} 2$ promedio por mes. Estas variaciones no tienen una relación directa con la generación de energía en el sistema fotovoltaico.

Comparando ambos arreglos fotovoltaicos se encontró también que, para el mismo periodo de tiempo, el arreglo con inversor centralizado presentó mayor cantidad de registros con cero energías generadas: 18 de los 335 registros; esta alta cantidad de registros cero podría indicar además que el arreglo con inversor centralizado es menos confiable, comparado con el arreglo con microinversores. Finalmente, para los once meses de prueba, la generación de energía total dada por el arreglo fotovoltaico con microinversores o con inversor centralizado cubrió los $499 \mathrm{kWh}$ anuales de consumo para un colombiano en el sector residencial. La generación total acumulada para el arreglo fotovoltaico con microinversores fue de $691 \mathrm{kWh}$ y para el arreglo con inversor centralizado fue de $593,2 \mathrm{kWh}$.

Se plantea la necesidad de estudiar el efecto de otros factores externos como el ángulo de inclinación de los paneles y el sombreado en la superficie del panel, o las emisiones de ceniza del volcán Nevado del Ruiz sobre la eficiencia del sistema fotovoltaico instalado en Manizales, como trabajo futuro.

\section{AGRADECIMIENTOS}

Los autores agradecen al Instituto de Estudios Ambientales-IDEA de la Universidad Nacional de Colombia, sede Manizales, por proveer los datos climatológicos de la estación meteorológica la Nubia. Además, agradecen el apoyo financiero al proyecto DIMA 39053 "Implementación y puesta en marcha de un Sistema de Generación Solar Fotovoltaica y Gestión Inteligente de Demanda" aprobado en la convocatoria nacional para el apoyo a proyectos de investigación y creación artística de la Universidad Nacional de Colombia 20172018, y al Programa Reconstrucción del Tejido Social en Zonas de Pos-conflicto en Colombia del Proyecto "Modelo ecosistémico de mejoramiento rural y construcción de paz. Instalación de capacidades locales". Financiado por Fondo Nacional de Financiamiento para la Ciencia, la Tecnología y la Innovación, Fondo Francisco José de Caldas, contrato No 213-2018, código 58960.

\section{REFERENCIAS}

[1] J. D. Marín-Jiménez, S. X. CarvajalQuintero, y J. M. Guerrero, "Island operation capability in the Colombian electrical market: a promising ancillary service of 
Análisis experimental del desempeño de un sistema solar fotovoltaico con inversor centralizado y con microinversores: caso de estudio Manizales

distributed energy resources," TecnoLógicas, vol. 21, no. 42, pp. 169-185, May. 2018. https://doi.org/10.22430/22565337.786

[2] A. G. Peralta-Sevilla y F. O. AmayaFernández, "Evolución de las redes eléctricas hacia Smart Grid en países de la Región Andina," Educ. en Ing., vol. 8, no. 15, pp. 4861, Jun. 2013. Disponible en: URL

[3] Y. A. Montoya-Mendoza, E. J. Ramírez, T. P. Pérez-Di Santis, L. M. Rujano-Molina, y N. A. Pérez-García, "Estado del arte de smart grid: parte I" Ing. al Día, vol. 2, no. 1, pp. 87107, Jan. 2016. Disponible en: URL

[4] Electric Power Research Institute, "Smart Grid Resource Center,” 2011. Disponible en: URL

[5] Unidad de Planeación Minero Energética UPME e Instituto de Hidrología, Meteorología y Estudio Ambientales "Atlas de Radiación Solar de Colombia," Ministerio de Minas y Energía - República de Colombia, Colombia, 2015. Disponible en: URL

[6] Procolombia, "Colombia y su potencial en fuentes de energía renovables," Colombia, 2018. Disponible en: URL

[7] Consejo Privado de Competitividad, "Informe Nacional de competitividad 2018-2019". Bogotá, 2018. Disponible en: URL

[8] Instituto de Hidrología Meteorologíay Estudios Ambientales - IDEAM, "Atlas Interactivo - Radiación IDEAM.” 2005. Disponible en: URL

[9] J. Gómez-Ramírez, J. D. Murcia-Murcia, y I. Cabeza-Rojas, "La Energía Solar Fotovoltaica en Colombia: potenciales, antecedentes y perspectivas," Universidad Santo Tomás, Bogotá, pp. 1-19. Oct. 2017. Disponible en: URL

[10] Unidad de Planeación Minero Energética UPME, "Plan de Expansión de Referencia Generación - Transmisión 2017 - 2031," Ministerio de Minas y Energía - República de Colombia, Colombia. Disponible en: URL

[11] J. Jiménez, J. E. Cardona, y S. X. Carvajal, "Location and optimal sizing of photovoltaic sources in an isolated mini-grid," TecnoLógicas, vol. 22, no. 44, pp. 61-80, Jan. 2019. https://doi.org/10.22430/22565337.1182

[12] A. Woyte, M. Richter, D. Moser, S. Mau, N. Reich, y U. Jahn, "Monitoring of Photovoltaic Systems: Good Practices and Systematic Analysis," en 28th European PV Solar Energy Conference and Exhibition, Paris, 2013, pp. 1-9. Disponible en: URL

[13] M. Ejgar y B. Momin, "Solar plant monitoring system: A review," en 2017 International Conference on Computing Methodologies and Communication (ICCMC), Erode, 2017, pp. 1142-1144. https://doi.org/10.1109/ICCMC.2017.8282652
[14] R. A. Messenger y J. Ventre, Photovoltaic Systems Engineering, 3a ed., Abingdon: Taylor \& Francis Group, 2010.

[15] K. Chari y A. Dessai, "Design of low power solar micro inverter for home application," Multidiscip. J. Res. Eng. Technol., pp. 74-79, Jun. 2015. Disponible en: URL

[16] R. Darussalam, R. I. Pramana, y A. Rajani, "Experimental investigation of serial parallel and total-cross-tied configuration photovoltaic under partial shading conditions," en 2017 International Conference on Sustainable Energy Engineering and Application (ICSEEA), Jakarta, 2017, pp. $140-144$. https://doi.org/10.1109/ICSEEA.2017.826769 9

[17] F. Muhammad, S. Sdeeq, y A. Ameen, "Effect of Series and Parallel Shading on the Photovoltaic Performance of Silicon Based Solar Panels," J. Technol. Innov. Renew. Energy, vol. 4, no. 4, pp. 152-156, Dec. 2015. https://doi.org/10.6000/1929-

6002.2015.04.04.5

[18] D. Murillo-Yarce, A. Alzate-Gómez, y A. Escobar-Mejía, "Análisis comparativo del control predictivo de corriente en convertidores VSI empleados en la conexión a red de energías renovables," TecnoLógicas, vol. 21 , no. 41 , pp. 45-62, 2018.

https://doi.org/10.22430/22565337.714

[19] M. H. Rashid, Electrónica de Potencia: circuitos, dispositivos y aplicaciones, $2^{\mathrm{a}}$ ed. Naucalpan de Juárez: Prentice Hall Hispanoamericana S.A, 1995.

[20] Solarpraxis, "Inverter and PV System Technology," Solarpraxis, 2011.

Disponible en: URL

[21] Solar Edge, "Performance of PV Topologies under Shaded Conditions," 2013.

Disponible en: URL

[22] P. Gevorkian, Solar Power Generation Problems, Solutions, and Monitoring, Cambridge: Cambridge University Press, 2016.

https://doi.org/10.1017/CBO9781316341414

[23] H. A. Sher y K. E. Addoweesh, "Microinverters - Promising solutions in solar photovoltaics", Energy Sustain. Dev., vol. 16, no. 4, pp. 389-400, Dec. 2012.

https://doi.org/10.1016/j.esd.2012.10.002

[24] D. M. Scholten, N. Ertugrul, and W. L. Soong, "Micro-inverters in small scale PV systems: A review and future directions", in 2013 Australasian Universities Power Engineering Conference (AUPEC), Hobart, 2013, pp. 1-6.

https://doi.org/10.1109/AUPEC.2013.6725465

GWL/Power Group, "GridFree Micro AC

Direct Inverter DC-AC 230V”, República 
Análisis experimental del desempeño de un sistema solar fotovoltaico con inversor centralizado y con microinversores: caso de estudio Manizales

Checa, 2017. Disponible en: URL

[26] I. F. Moran, J. A. Restrepo, M. L. OrozcoGutierrez, y J. M. Ramirez-Scarpetta, "Quasi-switched inverter using space vector pulse width modulation with triangular comparison for photovoltaic applications," TecnoLógicas, vol. 21, no. 42, pp. 95-110, May. 2018.

https://doi.org/10.22430/22565337.781

[27] T. Esram y P. L. Chapman, "Comparison of Photovoltaic Array Maximum Power Point Tracking Techniques," IEEE Transactions on Energy Conversion., vol. 22, no. 2, pp. 439449, Jun. 2007.

https://doi.org/10.1109/TEC.2006.874230

[28] Y. J. Mark Tung, A. Patrick Hu, y N. Kumar Nair, "Evaluation of Micro Controller Based Maximum Power Point Tracking Methods Using dSPACE Platform", en Australian University Power Engineering Conference, Melborne, 2006. Disponible en: URL

[29] D. P. Hohm y M. E. Ropp, "Comparative Study of Maximum Power Point Tracking Algorithms," Progress in photovoltaics: research and application, vol. 11, pp. 47-62, Nov. 2002. https://doi.org/10.1002/pip.459

[30] IEC System for Conformity Testing and Certification of Electrical Equipment, "Testing and measuring equipment/allowed subcontracting, Photovoltaic systems -Power conditioners-Procedure for measuring efficiency", IEC 61683:199, 2017. Disponible en: URL

[31] European Standards, CSN 50530 Overall efficiency of grid connected photovoltaic inverters, 2010. Disponible en: URL

[32] The Institute of Electrical and Electronics Engineers, IEEE Standard for Interconnection and Interoperability of Distributed Energy Resources with Associated Electric Power Systems Interfaces, New York, Feb. 2003. https://doi.org/10.1109/IEEESTD.2018.83321 12

[33] Componentes de acumulación, conversión y gestión de energía de sistemas fotovoltáicos. Calificación del diseño y ensayos ambientales, Norma NTC 5627:2008, 2008. Disponible en: URL

[34] Artefactos electrodomésticos y similares. Seguridad. Parte 1: requisitos generales, Norma NTC 2183:2014, 2014. Disponible en: URL

[35] A. Banshwar, N. K. Sharma, Y. R. Sood, and R. Shrivastava, "An international experience of technical and economic aspects of ancillary services in deregulated power industry: Lessons for emerging BRIC electricity markets", Renewable and Sustainable Energy Reviews, vol. 90, pp. 774-801, Jul.
2018.

https://doi.org/10.1016/j.rser.2018.03.085

[36] M. Braun et al., "Is the distribution grid ready to accept large-scale photovoltaic deployment? State of the art, progress, and future prospects," en 26th European Photovoltaic Solar Energy Conference and Exhibition (26th EU PVSEC), Hamburg, 2012, pp. 681-697.

https://doi.org/10.1002/pip.1204

[37] EvoEnergy, "Technical Requirements for the Connection of Inverter Energy (IES) Up To 30kVA,” 2018. Disponible en: URL

[38] TDK Components The Customer Magazine of TDK- EPC Corporation, "Effective protection of valuable solar installations," 2011. Disponible en: URL

[39] ABB solutions for solar energy- Low Voltage Productos, "Low Voltage Products - Solutions for solar energy," 2014. Disponible en: URL

[40] J. Flicker y J. Johnson, "Photovoltaic ground fault detection recommendations for array safety and operation," Sol. Energy, vol. 140, pp. 34-50, Dec. 2016.

https://doi.org/10.1016/j.solener.2016.10.017

[41] Comisión de Integración Energética Regional, "Guía para el uso de la Norma IEEE 1547," CIER, 2016. Disponible en: URL

[42] S. B. Kjaer, J. K. Pedersen, y F. Blaabjerg, "A Review of Single-Phase Grid-Connected Inverters for Photovoltaic Modules," IEEE Transactions on Industry Applications, vol. 41, no. 5, pp. 1292-1306, Sep. 2005. https://doi.org/10.1109/TIA.2005.853371

[43] Hybrytech, "YGE 60 Cell Series 2 HSF Smart," 2017. Disponible en: URL

[44] Instituto de Estudios Ambientales - IDEA, "Sistema Integrado de Monitoreo Ambiental de Caldas - SIMAC. Red de estaciones meteorológicas e hidrometeorológicas automáticas de Manizales. Estaciones para la gestión del riesgo ante desastres por deslizamientos. Primera Parte," Boletín Ambiental No. 145, Manizales, 2018.

Disponible en: URL

[45] Unimedios, "La U.N. alerta sobre nivel de lluvias en Manizales," Agencia de Noticias UN, Manizales, Oct. 2018. Disponible en: URL

[46] Instituto de Estudios Ambientales - IDEA, "Sistema Integrado de Monitoreo Ambiental de Caldas - SIMAC. Red de estaciones meteorológicas e hidrometeorológicas automáticas de Manizales. Estaciones para la gestión del riesgo ante desastres por deslizamientos. Segunda Parte," Boletín Ambiental No. 146, Colombia, 2018.

Disponible en: URL

[47] J. Romero, "Análisis del funcionamiento de 
Análisis experimental del desempeño de un sistema solar fotovoltaico con inversor centralizado y con microinversores: caso de estudio Manizales

paneles fotovoltaicos y su utilización en las regiones de la costa y sierra del Ecuador. Caso de estudio: Biblioteca Pompeu Fabra de Mataró," (Tesis de maestría), Universidad Politècnica de Catalunya, Barcelona, 2015. Disponible en: URL

[48] Enphase Energy, "Microinversor M250 de Enphase", 2016. Disponible en: URL

[49] Hersic International, "Energía Sostenible Con Calidad", 2018. Disponible en: URL

[50] Inc Enphase Energy, "Enphase MyEnlighten Web Application,” 2019.

Disponible en: URL

[51] F. S. Gazis, G. A. Vokas, I. J. Katsimardou, y J. K. Kaldelis, "Micro inverters for PV plants compared to the ordinary string or central inverters," en Conference for International Synergy in Energy, Environment, Tourism and contribution of Information Technology in Science, Economy, Society and Education, Grece, 2013. pp. 1-9. Disponible en: URL

[52] M. Johns, H.-P. Le, y M. Seeman, "GridConnected Solar Electronics," EE-290N-3Contemporary Energy Issues, no. Contemporary Energy Issues, pp. 1-12, 2009. Diponible en: URL

[53] Instituto de Estudios Ambientales - IDEAM, "Reporte Meteorológico," 2019.

Disponible en: URL

[54] A. C. Cortés Cortés, "Análisis de la variabilidad espacial y temporal de la precipitación en una ciudad de media montaña andina. Caso de estudio: Manizales" (Tesis de maestría). Universidad Nacional de Colombia, Departamento de Arquitectura y Urbanismo. Manizales Colombia, 2010. Disponible en: URL

[55] Alcaldía de Manizales, "Plan de Ordenamiento Territorial de Manizales 2017 - 2031," 2017. Disponible en: URL

[56] Federación Nacional de Cafeteros de Colombia y Centro Nacional de Investigaciones de Café "Pedro Uribe Mejía" CENICAFÉ, "Anuario Meteorológico Cafetero 2017”. Chinchiná: FNC y CENICAFÉ, 2018.

[57] R. Boukenoui, R. Bradai, A. Mellit, M.
Ghanes, y H. Salhi, "Comparative analysis of P\&amp;O, modified hill climbing-FLC, and adaptive P\&amp;O-FLC MPPTs for microgrid standalone PV system", en 2015 International Conference on Renewable Energy Research and Applications (ICRERA), Palermo, 2015. pp. 1095-1099. https://doi.org/10.1109/ICRERA.2015.741857 9

[58] K. L. Lian, J. H. Jhang, and y I. S. Tian, "A Maximum Power Point Tracking Method Based on Perturb-and-Observe Combined With Particle Swarm Optimization", IEEE Journal of Photovoltaics, vol. 4, no. 2, pp. 626-633, Jan. 2014. https://doi.org/10.1109/JPHOTOV.2013.2297 513

[59] O. Guenounou, B. Dahhou y F. Chabour, "Adaptive fuzzy controller based MPPT for photovoltaic systems," Energy Conversion and Management., vol. 78, pp. 843-850,

Feb. 2014. https://doi.org/10.1016/j.enconman.2013.07.0 93

[60] A. F. Murtaza, M. Chiaberge, F. Spertino, U. Tabrez Shami, D. Boero y M. De Giuseppe, "MPPT technique based on improved evaluation of photovoltaic parameters for uniformly irradiated photovoltaic array," Electric Power Systems Research, vol. 145, pp. 248-263, Apr. 2017. https://doi.org/10.1016/j.epsr.2016.12.030

[61] P. Kofinas, S. Doltsinis, A. I. Dounis y G. A. Vouros, "A reinforcement learning approach for MPPT control method of photovoltaic sources," Renewable Energy, vol. 108, pp. 461-473, Aug. 2017. https://doi.org/10.1016/j.renene.2017.03.008

[62] Instituto de Estudios Ambientales - IDEA, Universidad Nacional de Colombia "Boletín climatológico Trimestral - tercer trimestre de 2018," Sistema integrado de monitoreo ambiental de caldas -Simac, Colombia, Boletin, 2018. Disponible en: URL 